\title{
TWO-STAGE DISCRETE MECHANICAL MODELIN THREE-DIMENSIONAL SPACEFOR GARMENTSIMULATION
}

\author{
Xuan Luo ${ }^{1, *}$, Gaoming Jiang ${ }^{1,2}$,Honglian Cong ${ }^{1,3}$ \\ ${ }^{1}$ Engineering Research Center for Knitting Technology, Ministry of Education, Jiangnan University, Wuxi, China; \\ ${ }^{2}$ International Joint Research Laboratory for Novel Knitting Structural Materials at Jiangnan University, Wuxi, China; \\ ${ }^{3}$ Key Laboratory of Eco-textiles, Ministry of Education, Wuxi, China; \\ *Corresponding Authors:653011306@qq.com;
}

\begin{abstract}
:
This paper focuses on the better performance between the garment simulation result and the simulation speed. For simplicity and clarity, a notation "PART" is defined to indicate the areas between the garment and the human body satisfying some constraints. The discrete mechanical model can be achieved by the two-stage process. In the first stage, the garment can be divided into several PARTs constrained by the distance. In the second stage, the mechanical model of each PART is formulated with a mathematical expression. Thus, the mechanical model of the garment can be obtained. Through changing the constrained distance, the simulation result and the simulation speed can be observed. From the variable distance, a desired value can be chosen for an optimal value. The results of simulations and experiments demonstrate that the better performance can be achieved at a higher speed by saving runtime with the acceptable simulation results and the efficiency of the proposed scheme can be verified as well.
\end{abstract}

\section{Keywords:}

Garment simulation; Mechanical model; distance constraints

\section{Introduction}

Undoubtedly, clothes and the derivative garments play an essential role in the daily routine. How to simulate the realistic fabric appearance with the computer is a hot topic widely researched in the computer graphics in recent years. With the emergence of the virtual network environment, several fields, such as virtual fitting and online games, increasingly require the three-dimensional clothing simulation. In view of this, to achieve the virtual presentation of three-dimensional garments, it is necessary to establish a calculation model with high calculating efficiency and favorable simulation effects [12].

The three-dimensional garment simulation modeling can be roughly divided into two groups. One is the garment simulation based on the mesh, which applies mesh to represent garments, establishes the mechanical model to garments, and makes the garment produce the authentic deformation and wrinkles, so as to obtain the garments with the third dimension [3-4]. The other one is the garment simulation based on the yarn structure, which applies yarn as the elementary unit to construct and present fabrics to reflect the organization, structure, and characteristics of fabrics and represents the fabric appearance more realistically, thereby realizing the fabric deformation based on the interaction between yarns [5-6]. In this article, the first method mentioned is applied to garment simulation.

For such a study, there are numerous researchers who have proposed different ways for garment simulation. Provot [7] proposed mass-spring model, which describes the relationship between the clothing and the human body. This method applies
Hooke's law of relationship and can be described with an equation in Newton's second law. During solving an equation regarding the above statement, the position and velocity of the mass can be computed with numerical values. In this model, the spring is assumed to be linear, which exerts an influence on the accuracy of the simulation when overstretching of the spring occurs. Terzopoulos [8] proposed the continuum elastic deformation model based on Newton's law and the theory of elasticity. Carigan [9] further improved Terzopoulos' method and proposed the continuum elastic deformation model, but the computation of this algorithm is so complicated that it can hardly be used in the real-time simulation. Volino [10] proposed the accurate mass-spring model applicable to triangular meshes. In order to accurately model the anisotropic nonlinearity of fabrics, the polynomial-spline curve was used to approximately express the actually measured fabric attributes. Baraff [11] et al. established the garment model with triangular meshes, concluded both expansible deformation and shearing deformation within the triangular mesh, represented bending deformation through two adjacent triangular meshes with a common edge, and additionally maintained the simulation stability with the long-radius implicit integration algorithm. However, the model proposed was difficult to meet the realtime requirements. Hadap [12] adopted the rough mesh model to simulate the numerical integration and improved the speed of clothing simulation. At the same time, he also applied the geometric method to produce more subtle wrinkles, so as to greatly reduce the calculated amount. Choi [13] proposed the immediate buckling model based on Baraff's method. This model demonstrates abundant folds of the garment with high accuracy, but it requires more computation so that it is hard to meet the requirements of garment simulation in the real-time 
mode. Kang [14] et al. first established the physical model to the garment to obtain the key nodes in the model structure, and then they carried out the geometric processing and sampling curve interpolation based on the key nodes obtained, so as to acquire the skeleton nodes and internal nodes. As a result, the validity of garment simulation was effectively improved. Cordier and Liu [15-16] divided the garment into three types of areas using multiple models, that is, tight, loose, and floating areas. Different types of area are related to different models. This method can improve the accuracy of the simulation to some extent alongside its complexity. Jian Dong Yang [17] solved the corresponding equations with the fourth-order RungeKutta method; the inherent high computation of the fourthorder Runge-Kutta method is still unsolved. Matthias Muller et al [18] proposed the position-based dynamic method by which the apical position was directly operated and no other forces were analyzed but gravity. The constraint was used to represent the internal structure of garments and deal with collision. Although this method could demonstrate the basic changes on wrinkles and overhang of the cloth, it was unable to show the subtle wrinkles of the cloth without force analysis. Dongyong Zhu [19] segmented the human body, and different segmentation models were deduced based on the different parts of the human model, which requires more computation. As for Clemens Gro $\beta$ [20], the method is based on the idea of geometric prepositioning and physically based simulation for end-positioning. The geometric prepositioning algorithm automatically and simultaneously processes several pieces of clothing around the human body in such a way that the final fitting can be computed efficiently by a physically based cloth simulation. Thomaszewski [21] applied the finite element subdivision method into the garment simulation, but the simulation efficiency is relatively low because of the overloaded amount of calculation.

Although the desired simulation results can be achieved by the above methods in the first type of garment simulation, the simulation speed is unsatisfactory because of the solution of many differential equations. As a result, the above methods are not applicable in real-time occasions such as virtual try-on on the Internet because of the requirement of more time.

In view of the problem, this paper aims to improve the simulation speed as much as possible with acceptable results. For this, a two-stage mechanical model of the garment in a threedimensional space is proposed with general mathematical expressions. This paper makes the following contributions:

1. Define the concept "PART" and the corresponding distance constraints. To speed up the simulation, it is reasonable to decrease the simulation accuracy slightly, leading to the decrease in the segments of the garment. Unlike the conventional methods, in this method, the garment is divided into different continuous areas, which are defined as PARTs in this article based on the distance constraints between the garment and the human body at the first stage of the proposed scheme. According to the lower and upper values of the distance constraints, the number and shape of PARTs of the garment vary correspondingly. Through the adjustment of the values, a better comprehensive performance between the simulation time and the simulation accuracy can be achieved.

2. A mechanical model in the $3 D$ space is proposed within a PART and its corresponding general mathematical expressions are derived as well. At the second stage, within a PART, a mechanical model is provided in consideration of gravity and other forces. According to this mechanical model, the general mathematical expressions of the displacements along three axes are derived, with which the deformation of the garment within this PART can be demonstrated. When all the PARTs are determined, the entire garment simulation is finished.

Five types of the garments are chosen to be simulated with the proposed scheme and three reference methods. The simulation results show that the proposed scheme can reduce the simulation time effectively with proper lower or upper values of the distance constraints when compared to other methods. Consequently, it can be verified that with the proposed scheme, a better comprehensive performance between the simulation time and the simulation time can be achieved.

\section{Model and algorithm}

From the above discussion, aiming to obtain the better comprehensive performance of the simulation results and the simulation speed, the two-stage discrete mechanical model is proposed. The proposed scheme can perform in two stages. In the first stage, the distance between the garment and the human body is expressed in advance in a form of lower values and upper values. For simplicity and clarity, a concept "PART" of the garment is defined, which refers to the continuous area within a distance set, that is, the distances of all the discrete points in the PART are larger than the lower value but smaller than the upper value of the distance set. With the distance sets, the whole garment can be divided into different PARTs in accordance with the values in the distance sets. Then, the second stage is carried out. In a specific PART, it can be formulated as a mechanical model that can be expressed with force equations composed of gravity and other forces in a general mathematical form. With the mechanical model in a specific PART, the displacements of internal discrete points within this PART can be computed. In this manner, the deformation of the garment can be demonstrated when the displacements of all PARTs are determined. By slightly adjusting the distance constraints, the better performance between the simulation accuracy and the simulation speed can be achieved as well.

To provide a clear explanation, the symbols with the descriptions are listed in Table 1. It is noted that the symbols in Table 1 indicate a continuous state.

\subsection{First stage algorithm}

In the first stage, the definition of "PART" is given. According to this concept, the whole garment can be divided into several PARTs, with which the simulation can be speeded up with acceptable simulation results. 
Table 1. Symbols and description

\begin{tabular}{|c|c|}
\hline Symbols & Description \\
\hline$g_{f}(x, y, z)$ & Mass density of the garment \\
\hline$q_{f}(x, y, z)$ & $\begin{array}{l}\text { Force density of the human body on the } \\
\text { garment }\end{array}$ \\
\hline$p_{f}(x, y, z)$ & Internal line force coefficient of the garment \\
\hline$q(x, y, z)$ & Curved surface equation of the human body \\
\hline$p(x, y, z)$ & Curved surface equation of the garment \\
\hline$\alpha_{q}(x, y, z)$ & $\begin{array}{l}\text { Angle of normal vector of } \\
\qquad x \text {-axis }\end{array}$ \\
\hline$\beta_{q}(x, y, z)$ & $\begin{array}{l}\text { Angle of normal vector of } q(x, y, z) \text { and } \\
y \text {-axis }\end{array}$ \\
\hline$\gamma_{q}(x, y, z)$ & $\begin{array}{l}\text { Angle of normal vector of } q(x, y, z) \text { and } \\
z \text {-axis }\end{array}$ \\
\hline$\alpha_{p}(x, y, z)$ & $\begin{array}{l}\text { Angle of normal vector of } p(x, y, z) \text { and } \\
x \text {-axis }\end{array}$ \\
\hline$\beta_{p}(x, y, z)$ & $\begin{array}{l}\text { Angle of normal vector of } p(x, y, z) \text { and } \\
y \text {-axis }\end{array}$ \\
\hline$\gamma_{p}(x, y, z)$ & $\begin{array}{l}\text { Angle of normal vector of } p(x, y, z) \text { and } \\
z \text {-axis }\end{array}$ \\
\hline
\end{tabular}

\subsubsection{PART definition}

In this paper, "PART" is defined as a continuous area satisfying some constraints of the distance between the garment and the human body.

In the following part, the general expressions and formulations are based on the PART concept. To give a clear explanation of the proposed method, some symbols and the corresponding notions within the $k$ th PART are defined in Table 2, where the symbols marked with " $k$ " represent the variables for the $k$ th PART.

Specifically, for the $k$ th PART, the corresponding distance set $S_{k}$ can be expressed with a form in equation (1). In this equation, $k$ represents the index of the PART, $\|\cdot\|_{2}$ denotes 2-norm, $V_{L}^{(k)}$ is the predefined value for the lower range, and $V_{H}^{(k)}$ refers to the upper range, and $x_{k, i_{0}}, y_{k, i_{1}}$, and $z_{k, i_{2}}$ refer to the discrete coordinates for the garment.

$V_{L}^{(k)} \leq \underset{S_{k}}{\arg } \min _{\left(x_{k, i_{0}}, y_{k, i_{1}}, z_{k, i_{2}}\right) \in S_{k}}\left\|p\left(x_{k, i_{0}}, y_{k, i_{1}}, z_{k, i_{2}}\right)-q(x, y, z)\right\|_{2} \leq V_{H}^{(k)}$

It can be derived that the size of the set $S_{k}$ is dependent on the number of the coordinate pairs $\left(x_{k, i_{0}}, y_{k, i_{1}}, z_{k, i_{2}}\right)$, which satisfies the constraints in (1). At the same time, the
Table 2.Variables and notations for the $k$ th PART

\begin{tabular}{|c|c|}
\hline Variables & Notations \\
\hline$V_{L}^{(k)}$ & Lower preset value \\
\hline$V_{H}^{(k)}$ & Upper preset value \\
\hline$S_{k}$ & The set of the coordinate pairs \\
\hline$F_{\bullet}^{(k, 0)}$ & $\begin{array}{c}\text { The gravity force along } \bullet \text { axis. }{ }^{\bullet} \text { represents } \\
\qquad x, y \text {, or } z\end{array}$ \\
\hline$F_{\bullet}^{(k, 1)}$ & $\begin{array}{l}\text { The anti-force from the human body along } \\
\bullet \text { axis }\end{array}$ \\
\hline$F_{\bullet}^{(k, 2)}$ & $\begin{array}{l}\text { The force from adjacent PARTs along } \bullet \\
\text { axis }\end{array}$ \\
\hline$F^{(k)}$ & The resultant force along $\bullet$ axis \\
\hline$F^{(k)}$ & The resultant force for the $k$ th PART \\
\hline$D x^{(k)}$ & The displacement along $x$-axis \\
\hline$D y^{(k)}$ & The displacement along $y$-axis \\
\hline$D z^{(k)}$ & The displacement along $z$-axis \\
\hline
\end{tabular}

number of the coordinate pairs is related to the distance range with the lower value $V_{L}^{(k)}$ and the upper value $V_{H}^{(k)}$. Consequently, the lower value and the upper value, $V_{L}^{(k)}$ and $V_{H}^{(k)}$, have an influence on the size of the PARTs.

\subsubsection{Division of the garment}

On the basis of the "PART" concept and the distance set, the garment can be divided into several PARTs as shown in Figure 1. Different from the methods using the mesh division based on triangles and polygons, the shapes of PARTs are different, which can also be observed in the figure.

As stated earlier, the lower value and the upper value of the distance set, $V_{L}^{(k)}$ and $V_{H}^{(k)}$, can influence the size of the PARTs. For example, by increasing the upper value $V_{H}^{(k)}$ or by lowering the lower value $V_{L}^{(k)}$, the size of the $k$ th PART can be expanded and the number of the PARTs of the garment can be reduced. Given that the PART of the garment contains several triangles and polygons in general, proper lower and upper values of the distance set can make that the number of the PARTs is smaller than that of triangles and polygons of the garment. Thus, the simulation time can be saved.

\subsection{Second stage algorithm}

In the second algorithm, a mechanical model of one of the PARTs of the garment in 3D space for the $k$ th PART is illustrated in Figure 2, considering the gravity and other forces, where $z$-axis is in the opposite direction of the gravity and $x$-axis and $y$-axis constitute a plane perpendicular to $z$-axis. 


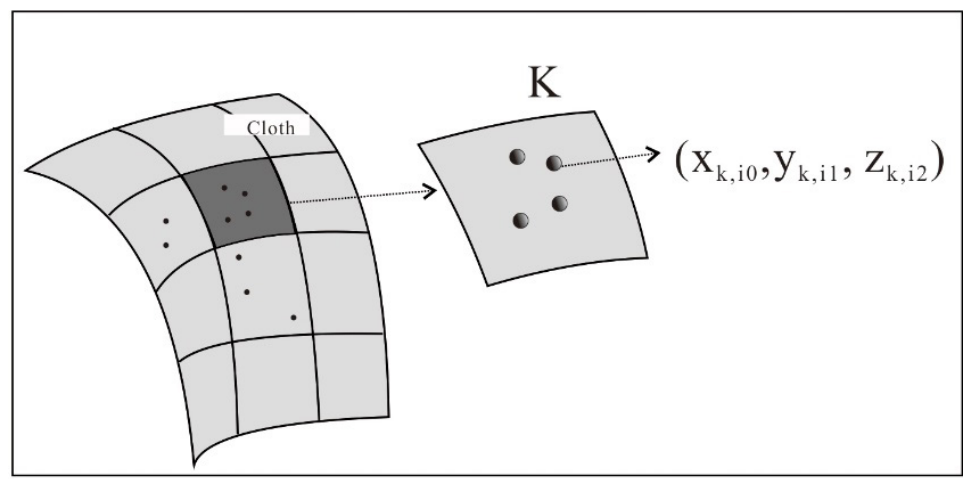

Figure 1 PART demonstration of the garment

To simplify the derivation, the same displacement along one axis is assumed. Let $D x^{(k)}, D y^{(k)}$, and $D z^{(k)}$ represent the displacement of the $k$ th PART along the $x$-axis, $y$-axis, and $z$-axis, respectively.

For the computer simulation of the algorithm, the expressions in Table 1 in a continuous state should be rewritten in the discrete form within each PART. Specifically, for the $k$ th PART, the coordinate pairs in this PART constitute a set denoted as $S_{k}$ and the coordinate pairs in the set $S_{k}$ are represented by $\left(x_{k, i_{0}}, y_{k, i_{1}}, z_{k, i_{2}}\right)$, where $\left(x_{k, i_{0}}, y_{k, i_{1}}, z_{k, i_{2}}\right) \subset S_{k}, x_{k, i_{0}}$ stands for the $i_{0}$ th component along the $x$-axis, $y_{k, i_{1}}$ is for the $i_{1}$ th component along the $y$-axis, and $z_{k, i_{2}}$ is for the $i_{2}$ th component along z-axis. Accordingly, the discrete formulation relating to the expressions in Table 1 can be determined by substituting the continuous variables with the corresponding discrete variables directly. For example, for the curved surface equation of the garment, $p(x, y, z)$, the corresponding discrete form in the $k$ th PART becomes expressible with $p\left(x_{k, i_{0}}, y_{k, i_{1}}, z_{k, i_{2}}\right)$ . In a similar manner, the discrete expressions relating to other continuous functions can be determined. In the following demonstration, a general expression of the mechanical model can be derived by the discrete forms.

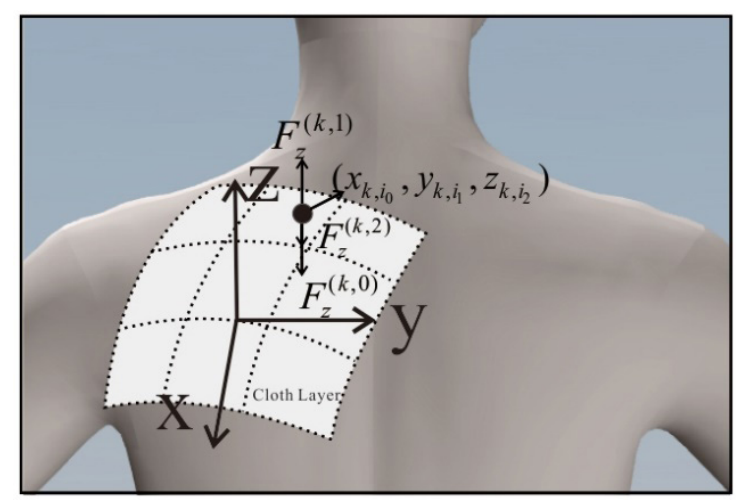

Figure 2. Mechanical model in 3D space.

The gravity within the $k$ th PART, $F_{z}^{(k, 0)}$, can be calculated by the summation of the gravity components at the point $\left(x_{k, i_{0}}, y_{k, i_{1}}, z_{k, i_{2}}\right)$ from the corresponding set $S_{k}$ of the $k$ th PART. The gravity component at the point $\left(x_{k, i_{0}}, y_{k, i_{1}}, z_{k, i_{2}}\right)$ is the multiplication of the gravity constant $g(=9.8 \mathrm{~N} / \mathrm{kg})$ and the mass of this component. Besides, it is known that the mass is the multiplication of the mass density and the volume. The mass density at this point in the discrete form can be represented by $g_{f}\left(x_{k, i_{0}}, y_{k, i_{1}}, z_{k, i_{2}}\right)$, which is derived from the continuous expression in Table $1, g_{f}(x, y, z)$, through the use of the above method. To simplify the computation, the shape at this point can be regarded as a cuboid. As a result, the volume at this point can be calculated by using the length, width, and height, which can be evaluated by the difference of the coordinates between this point and the adjacent point along three axes. That is to say, $x_{k, i_{0}+1}-x_{k, i_{0}} y_{k, i_{1}+1}-y_{k, i_{1}}$ and $z_{k, i_{2}+1}-z_{k, i_{2}}$ represent the length, width, and height of the cuboid, respectively. Therefore, all the gravity components constitute the gravity in the $k$ th PART, $F_{z}^{(k, 0)}$ with the form as shown in equation (2).

The anti-force from the human body along $z$-axis within the $k$ th PART, $F_{z}^{(k, 1)}$ refers to the force component along $z$-axis of the total force of the human body on the garment. To simplify the discussion, the garment is assumed to be directly and closely attached to the human body within this PART, that is, in this PART, the curved surface function of the garment is the same as that of the human body. As a result, the discrete form of the function is described as $q\left(x_{k, i_{0}}, y_{k, i_{1}}, z_{k, i_{2}}\right)$, which is derived from its continuous expression of the curved surface $q(x, y, z)$ using the abovementioned method. For the continuous curved surface expression $q(x, y, z)$, one can measure the angle between the normal vector of the surface and the $z$-axis with the partial differential equation along three axes, that is, $\partial q / \partial x$, $\partial q / \partial y$ and $\partial q / \partial z$, where $q$ is the abbreviation for $q(x, y, z)$. Consequently, the angle of the normal vector along $z$-axis, ${ }^{\gamma_{q}}$, can be determined at the point $\left(x_{k, i_{0}}, y_{k, i_{1}}, z_{k, i_{2}}\right)$ from the corresponding set $S_{k}$ by replacing the continuous variables with the discrete variables as shown in equation (3). Then, the anti-force of the human body on the garment in the $k$ th PART will be computed. According to Table 1, the force density of the human body on the garment has been assumed. Similar to the calculation of the gravity within the $k$ th PART, $F_{z}^{(k, 0)}$, the anti-force component at the point $\left(x_{k, i_{0}}, y_{k, i_{1}}, z_{k, i_{2}}\right)$ is the multiplication of the force density of the human body on the garment $q_{f}\left(x_{k, i_{0}}, y_{k, i_{1}}, z_{k, i_{2}}\right)$ and the volume at this point. The volume can be simplified with the difference of thecoordinates between this point and the adjacent point along three axes, that is, $x_{k, i_{0}+1}-x_{k, i_{0}}, y_{k, i_{1}+1}-y_{k, i_{1}}$, and $z_{k, i_{2}+1}-z_{k, i_{2}}$. After this, the total anti-force at this point can be determined by $q_{f}\left(x_{k, i_{0}}, y_{k, i_{1}}, z_{k, i_{2}}\right)\left(x_{k, i_{0}+1}-x_{k, i_{0}}\right) \cdot\left(y_{k, i_{1}+1}-y_{k, i_{1}}\right) \cdot\left(z_{k, i_{2}+1}-z_{k, i_{2}}\right)$. The anti-force component along $z$-axis is related to the total anti-force and the angle $\gamma_{q}$ at the point $\left(x_{k, i_{0}}, y_{k, i_{1}}, z_{k, i_{2}}\right)$ The equivalent anti-force in the $k$ th PART $F_{z}^{(k, 1)}$ can be 
determined by the summation of the force components along the $z$-axis at all points with the form in equation (2).

The force from the adjacent PARTs is closely connected with garment materials and the displacement of the materials. In practice, this type of force is treated as the spring model [7]. To simplify the analysis, the internal line force coefficients function $p_{f}(x, y, z)$ in the continuous form is defined to differentiate the garment materials in Table 1. As a spring model, the equivalent force from adjacent PARTs within the $k$ th PART, $F_{z}^{(k, 2)}$ can be computed with the equivalent coefficient of elasticity and the displacement along the $z$-axis. With the above assumptions, the displacement along $z$-axis, $D z^{(k)}$ is identical within the same PART. Under this circumstance, the equivalent coefficient of elasticity is crucial to the determination of the force from the adjacent PARTs. To calculate the equivalent coefficient of elasticity, the following variables should be determined: the discrete form of the internal line force coefficient function $p_{f}(x, y, z)$ and the angle between the normal vector of the curved surface equation of the garment $p(x, y, z)$ and the $z$-axis $\gamma_{p}$. The discrete form of the internal line force coefficient function at the point $\left(x_{k, i_{0}}, y_{k, i_{1}}, z_{k, i_{2}}\right)$ can be derived from its corresponding expression by replacing the continuous variables with the discrete variables, that is, $p_{f}\left(x_{k, i_{0}}, y_{k, i_{1}}, z_{k, i_{2}}\right)$. For the angle $\gamma_{p}$, it can be computed in a similar way as the calculation of the angle $\gamma_{q}$. As a result, the angle $\gamma_{p}$ can be obtained by the partial differential equation of the curved surface equation of the garment along three axes at the point $\left(x_{k, i_{0}}, y_{k, i_{1}}, z_{k, i_{2}}\right)$ as shown in equation (4), that is, $\partial p / \partial x, \partial p / \partial y$, and $\partial p / \partial z$.

$$
\begin{aligned}
& F_{z}^{(k, 0)=} \sum_{\left(x_{k, i_{0}}, y_{k, i_{1}}, z_{k, i_{2}}\right) \subset S_{k}} g \cdot g_{f}\left(x_{k, i_{0}}, y_{k, i_{1}}, z_{k, i_{2}}\right) \cdot\left(x_{k, i_{0}+1}-x_{k, i_{0}}\right) \cdot \\
& \quad\left(y_{k, i_{1}+1}-y_{k, i_{1}}\right) \cdot\left(z_{k, i_{2}+1}-z_{k, i_{2}}\right) \\
& F_{z}^{(k, 1)}=\sum_{\left(x_{k, i_{0}}, y_{k, i_{1}}, z_{k, i_{2}}\right) \subset S_{k}} q_{f}\left(x_{k, i_{0}}, y_{k, i_{1}}, z_{k, i_{2}}\right) \cdot \cos \left(\gamma_{q}\right) \cdot \\
& \left(x_{k, i_{0}+1}-x_{k, i_{0}}\right) \cdot\left(y_{k, i_{1}+1}-y_{k, i_{1}}\right) \cdot\left(z_{k, i_{2}+1}-z_{k, i_{2}}\right) \\
& F_{z}^{(k, 2)}=D z^{(k)} \cdot \sum_{\left(x_{k, i_{0}}, y_{k, i_{1}}, z_{k, i_{2}}\right) \in S_{k}} p_{f}\left(x_{k, i_{0}}, y_{k, i_{1}}, z_{k, i_{2}}\right) \cdot \cos \left(\gamma_{p}\right) \cdot \\
& q\left(x_{k, i_{0}}, y_{k, i_{1}}, z_{k, i_{2}}\right) \cdot\left(x_{k, i_{0}+1}-x_{k, i_{0}}\right) \cdot\left(y_{k, i_{1}+1}-y_{k, i_{1}}\right) \cdot \\
& \left(z_{k, i_{2}+1}-z_{k, i_{2}}\right)
\end{aligned}
$$

$$
\begin{aligned}
& \mathbf{e}_{q}=\left(\frac{\partial q}{\partial x}, \frac{\partial q}{\partial y}, \frac{\partial q}{\partial z}\right) \\
& \gamma_{q}=\left.\arccos \left(\frac{\partial q / \partial z}{\left\|e_{q}\right\|_{2}}\right)\right|_{z=, z_{k, i_{2}}} ^{x}=x_{k, i_{0}} \\
& y=y_{k, i_{1}} \\
& \mathbf{e}_{p}=\left(\frac{\partial p}{\partial x}, \frac{\partial p}{\partial y}, \frac{\partial p}{\partial z}\right) \\
& \gamma_{p}=\left.\arccos \left(\frac{\partial p / \partial z}{\left\|e_{p}\right\|_{2}}\right)\right|_{z=} ^{x}=x_{k, i_{0}} \\
& y=y_{k, i_{1}}
\end{aligned}
$$

To make the $k$ th PART stable, it is required that the force balance along $z$-axis and the corresponding mathematical expression should be determined, as shown in equation (5).

$$
F_{z}^{(k, 1)}-F_{z}^{(k, 0)}-F_{z}^{(k, 2)}=0
$$

To calculate the displacement along z-axis, $D z^{(k)}$, let $F_{z}^{(k, a)}$ represent the second item on the right side of the equal sign of the $F_{z}^{(k, 2)}$ in equation (2), that is,

$$
\begin{aligned}
& F_{z}^{(k, a)}=\sum_{\left(x_{k, i_{0}}, y_{k, i_{1}}, z_{k, i_{2}}\right) \subset S_{k}} p_{f}\left(x_{k, i_{0}}, y_{k, i_{1}}, z_{k, i_{2}}\right) \cdot \cos \left(\gamma_{p}\right) \cdot \\
& q\left(x_{k, i_{0}}, y_{k, i_{1}}, z_{k, i_{2}}\right) \cdot\left(x_{k, i_{0}+1}-x_{k, i_{0}}\right) \cdot\left(y_{k, i_{1}+1}-y_{k, i_{1}}\right) . \\
& \left(z_{k, i_{2}+1}-z_{k, i_{2}}\right)
\end{aligned}
$$

From equation (5), it can be obtained that

$$
F_{z}^{(k, 2)}=F_{z}^{(k, 1)}-F_{z}^{(k, 0)}
$$

Substituting $(2)$ and $F_{z}^{(k, a)}$ into (5), the displacement along z-axis, $D z^{(k)}$ can be computed in equation (6) as follows.

$$
D z^{(k)}=\frac{F_{z}^{(k, 1)}-F_{z}^{(k, 0)}}{F_{z}^{(k, a)}}
$$

As assumed earlier, z-axis is in the opposite direction of the gravity, $x$-axis and $y$-axis constitute a plane perpendicular to the $z$-axis. It can be deduced that there is no gravity component along $x$ - and $y$-axes, that is, the gravity components along $x$ and $y$-axes, $F_{x}^{(k, 0)}$ and $F_{y}^{(k, 0)}$, are zero. The force balance equations along $x$ - and $y$-axes are similar to those along $z$-axis in equation (5) by setting gravity components to zero and the corresponding force equations can be derived from the form

$$
\begin{aligned}
& F_{x}^{(k, 1)}-F_{x}^{(k, 2)}=0 \\
& F_{y}^{(k, 1)}-F_{y}^{(k, 2)}=0
\end{aligned}
$$

Similar to the calculation of the displacement along z-axis, the displacements along $\mathrm{x}$-and y-axes, $D x^{(k)}$ and $D y^{(k)}$ , have similar expressions to those of $D z^{(k)}$ by setting the gravity components to zero as shown in equation (7). The corresponding expressions along $x$ - and $y$-axes such as $F_{x}^{(k, 1)}$ can be computed by replacing the angle $\gamma_{q}$ with the angle of the normal vector of the curved surface of the human body, $\alpha_{q}$ and $\beta_{q}$. For auxiliary variables, $F_{x}^{(k, a)}$ and $F_{y}^{(k, a)}$ can be also determined by replacing the angle with the angle of the normal vector of the curved surface of the garment, $\alpha_{p}$ and $\beta_{p}$ . Consequently, the displacements along $x$-and y-axes, $D x^{\left(k^{p}\right)}$ and $D y^{(k)}$, can be determined by the above parameters similar to $D z^{(k)}$ in equation (7). 


$$
\begin{aligned}
& D x^{(k)}=\frac{F_{x}^{(k, 1)}}{F_{x}^{(k, a)}} \\
& D y^{(k)}=\frac{F_{y}^{(k, 1)}}{F_{y}^{(k, a)}} \\
& F_{x}^{(k, 1)}=\sum_{\left(x_{k, 0_{0}}, y_{k, l_{1}}, z_{k, k_{2}}\right) \subset S_{k}} q_{f}\left(x_{k, i_{0}}, y_{k, i_{1}}, z_{k, i_{2}}\right) \cdot \cos \left(\alpha_{q}\right) \cdot \\
& \left(x_{k, i_{0}+1}-x_{k, i_{0}}\right) \cdot\left(y_{k, i_{1}+1}-y_{k, i_{1}}\right) \cdot\left(z_{k, i_{2}+1}-z_{k, i_{2}}\right) \\
& F_{x}^{(k, a)}=\sum_{\left(x_{k, i_{0}}, y_{k, j_{1}}, z_{k, j_{2}}\right) \subset S_{k}} p_{f}\left(x_{k, i_{0}}, y_{k, i_{1}}, z_{k, i_{2}}\right) \cdot \cos \left(\alpha_{p}\right) \cdot q\left(x_{k, i_{0}}, y_{k, i_{1}}, z_{k, i_{2}}\right) \text {. } \\
& \left(x_{k, i_{0}+1}-x_{k, i_{0}}\right) \cdot\left(y_{k, i_{1}+1}-y_{k, i_{1}}\right) \cdot\left(z_{k, i_{2}+1}-z_{k, i_{2}}\right) \\
& F_{y}^{(k, 1)}=\sum_{\left(x_{k, j_{0}}, y_{k, 1}, z_{k, i_{2}}\right)} \sum_{S_{k}} q_{f}\left(x_{k, i_{0}}, y_{k, i_{1}}, z_{k, i_{2}}\right) \cdot \cos \left(\beta_{q}\right) \cdot \\
& \left(x_{k, i_{0}+1}-x_{k, i_{0}}\right) \cdot\left(y_{k, i_{1}+1}-y_{k, i_{1}}\right) \cdot\left(z_{k, i_{2}+1}-z_{k, i_{2}}\right) \\
& F_{y}^{(k, a)}=\sum_{\left(x_{k, i_{0}}, y_{k, j}, z_{k, i_{2}}\right) \in S_{k}} p_{f}\left(x_{k, i_{0}}, y_{k, i_{1}}, z_{k, i_{2}}\right) \cdot \cos \left(\beta_{p}\right) \cdot q\left(x_{k, i_{0}}, y_{k, i_{1}}, z_{k, i_{2}}\right) \text {. } \\
& \left(x_{k, i_{0}+1}-x_{k, i_{0}}\right) \cdot\left(y_{k, i_{1}+1}-y_{k, i_{1}}\right) \cdot\left(z_{k, i_{2}+1}-z_{k, i_{2}}\right)
\end{aligned}
$$

In addition, the corresponding angles, $\alpha_{q}, \beta_{q}, \alpha_{p}$, and $\beta_{p}$, are expressed in equation (8) with the discrete form of the partial differential equation at the point $\left(x_{k, i_{0}}, y_{k, i_{1}}, z_{k, i_{2}}\right)$. The expressions of the angles the above parameters $\gamma_{q}$ and $\gamma_{p}$ in equations (3) and (4).

$$
\begin{gathered}
\alpha_{q}=\arccos \left(\frac{\partial q / \partial x}{\left\|e_{q}\right\|_{2}}\right) \mid \begin{array}{l}
x=x_{k, i_{0}} \\
y=y_{k, i_{1}} \\
z=, z_{k, i_{2}}
\end{array} \\
\beta_{q}=\arccos \left(\frac{\partial q / \partial y}{\left\|e_{q}\right\|_{2}}\right) \mid \begin{array}{l}
x=x_{k, i_{0}} \\
y=y_{k, i_{1}} \\
z=, z_{k, i_{2}}
\end{array} \\
\alpha_{p}=\arccos \left(\frac{\partial p / \partial x}{\left\|e_{p}\right\|_{2}}\right) \mid \begin{array}{l}
x=x_{k, i_{0}} \\
y=y_{k, i_{1}} \\
z=, z_{k, i_{2}}
\end{array} \\
\beta_{p}=\arccos \left(\frac{\partial p / \partial y}{\left\|e_{p}\right\|_{2}}\right) \mid \begin{array}{l}
x=x_{k, i_{0}} \\
y=y_{k, i_{1}} \\
z=, z_{k, i_{2}}
\end{array}
\end{gathered}
$$

In general, the displacements along three axes can be determined with equations from (2) to (8). For some special cases, for example, the garment is contactless with human body such as the bottom dress shown in Figure 3. In this case, there exists no anti-force from human body, that is, the direction of the force in the kth PART is along z-axis. The expressions of displacements along three axes are simplified in equation (9).

$$
\begin{aligned}
& D x^{(k)}=0 \\
& D y^{(k)}=0 \\
& D z^{(k)}=\frac{F_{z}^{(k, 0)}}{F_{z}^{(k, a)}}
\end{aligned}
$$

On the basis of the simplified form in equation (9) and Figure 3 , it can be derived that the force components from the human body are zero along $x$ - and $y$-axes. This means that there are no displacements from the human body along these two directions. The displacement along the $z$-axis is determined by the gravity and the boundary of the kth PART.

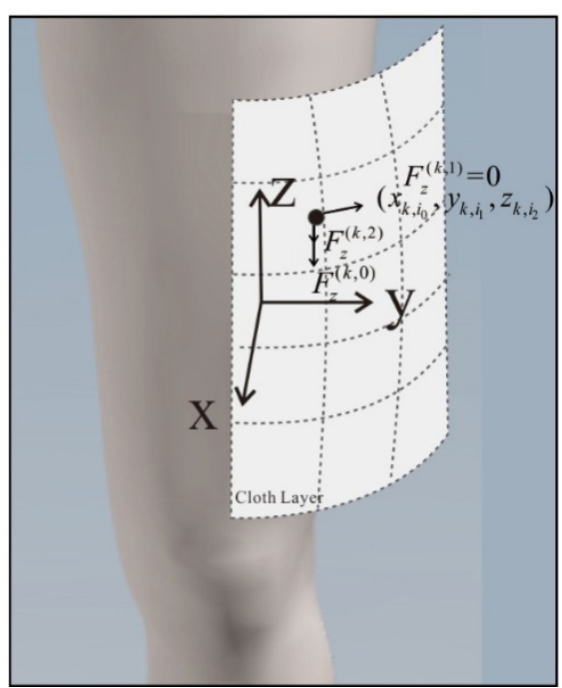

Figure 3. Garment which is contactless with the human body.

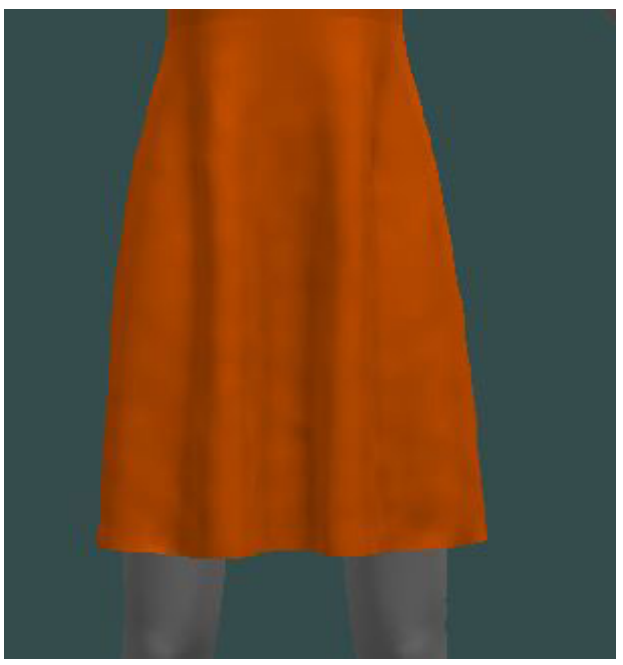

Figure 4. A demonstration of bottom dress in 3D space.

\section{Simulation and analysis}

The mechanical model proposed in this article is applied to dress simulation as an example to verify the performance. The simulation results and the simulation speed can be compared with those of other methods.

\subsection{PART determination with the distance constraints}

For the verification of the performance of the proposed scheme, different types of the garment were chosen for simulation. At the first stage, the distance range of each PART is to be determined. Generally speaking, in practice, it is very difficult to determine the range directly. To simplify the simulation process, the computer-aided method is applied. Specifically, the number of levels of range constraints and range values of each level are evaluated roughly. With the evaluated values, the lower range value and the upper range value of each level are adjusted slightly to obtain the simulation results. When the simulation 
results and speed can satisfy the requirement intuitively, one of the acceptable distance constraints is found. Otherwise, the above process is repeated. In this simulation, the number of the levels is set to 19 initially. With methods given above, the desired values of distance constraints are listed in Table 3, where the symbol " $\infty$ " represents infinity.

Table 3. Range values for the general expression of the distance

\begin{tabular}{|c|c|c|}
\hline Index & Lower range value & Upper range value \\
\hline 1 & 0 & 0.06 \\
\hline 2 & 0.06 & 0.085 \\
\hline 3 & 0.085 & 0.15 \\
\hline 4 & 0.15 & 0.25 \\
\hline 5 & 0.25 & 0.35 \\
\hline 6 & 0.35 & 0.45 \\
\hline 7 & 0.45 & 0.5 \\
\hline 8 & 0.5 & 1 \\
\hline 9 & 1 & 1.25 \\
\hline 10 & 1.25 & 1.75 \\
\hline 11 & 1.75 & 2 \\
\hline 12 & 2 & 2.5 \\
\hline 13 & 2.5 & 3 \\
\hline 14 & 3.5 & 4.5 \\
\hline 15 & 4.5 & 7 \\
\hline 16 & 7 & 9 \\
\hline 17 & 9 & 10 \\
\hline 18 & 10 & 11 \\
\hline 19 & 11 & $\infty$ \\
\hline
\end{tabular}

From the values presented in Table 3 and the distance constraints of a PART described in equation (1), the desired PARTs of the dress can be computed with the proposed scheme at the first stage, which is depicted in Figure 5. As shown in the figure, the shapes of the PARTs are different. Compared to the existing divisions of the dress such as triangles or polygons, the PARTs obtained with the proposed scheme can vary with the lower value and the upper value in Table 3. In general, decreasing the lower value or increasing the upper value of each level will expand the area of the PART. This means that the number of the PART for a garment will decrease, resulting in speeding up the simulation speed and lowering down the simulation accuracy. Therefore, a better balance between the simulation time and the simulation accuracy can be realized by properly adjusting the constraint values of levels.

\section{$\underline{3.2 \text { Dress simulation }}$}

In the second stage, the mechanical model within a PART can be calculated by referring to equations (2)-(9). On the basis of the expressions of the human body and the garment and equations (3), (4), and (8), the angles of the normal vector of the

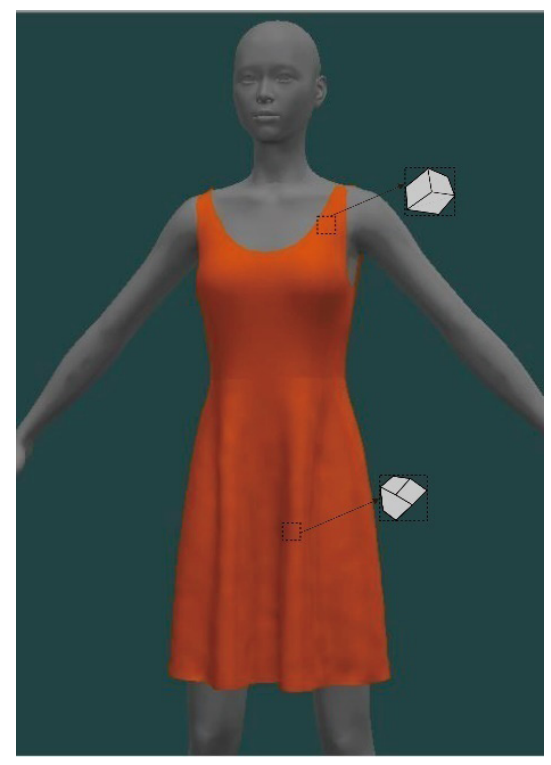

Figure 5. PART division using the proposed scheme.

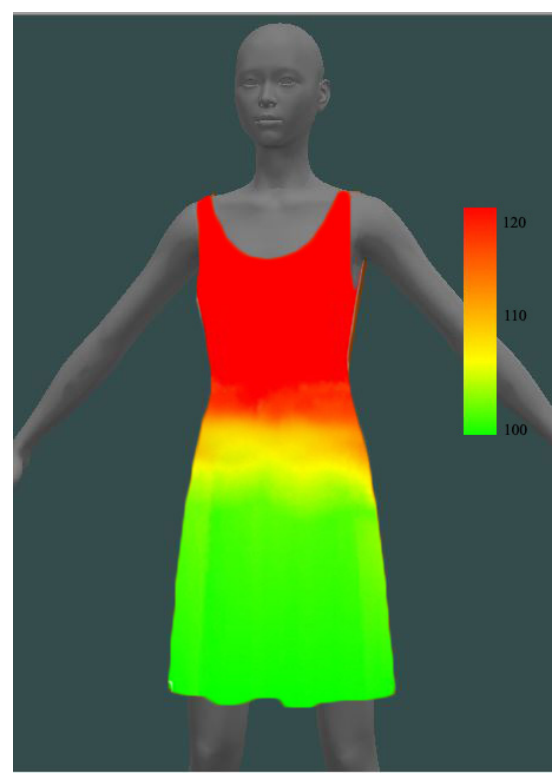

Figure 6. Force distribution of the dress

corresponding curved surface at the point $\left(x_{k, i_{0}}, y_{k, i_{1}}, z_{k, i_{2}}\right)$ within the $k$ th PART along three axes can be calculated. In this manner, the angles $\alpha_{q}, \beta_{q}, \gamma_{q}, \alpha_{p}, \beta_{p}$, and $\gamma_{p}$ can be computed. Substituting the above angles and the expressions of the human body and the garment into equations (6) and (7), the displacements and the force components along three axes can be determined.

As stated earlier, the force of each PART can be simplified to three components. And then, the resultant force of each PART can be simplified to the force components along three axes. For the force along $z$-axis, there are three force components, $F_{z}^{(k, 0)}, F_{z}^{(k, 1)}$, and $F_{z}^{(k, 2)}$. Therefore, the resultant force along z-axis $F_{z}^{(k)}$ is the summation of the three force components in this direction, that is, $F_{z}^{(k)}=F_{z}^{(k, 0)}+F_{z}^{(k, 1)}+F_{z}^{(k, 2)}$. In this way, the resultant forces along $x$ - and $y$-axis, $F_{x}^{(k)}$ and $F_{y}^{(k)}$, have the similar expression to that of $F_{z}^{(k)}$. It is noted that the difference among $F_{z}^{(k, 0)}, F_{x}^{(k, 0)}$, and $F_{y}^{(k, 0)}$ are usually zero as 
discussed earlier. For the $k$ th PART, the resultant force $F^{(k)}$ can be computed based on the force components along three axes with the form

$$
F^{(k)}=\sqrt{\left(F_{x}^{(k)}\right)_{2}+\left(F_{y}^{(k)}\right)^{2}+\left(F_{z}^{(k)}\right)^{2}}
$$

To observe the resultant forces of the dress intuitively, a color demonstration with legend is applicable, which is shown in Figure 6. The colors represent the strength of the forces. Specifically, the color located at the upper position of the legend indicates the strength is larger than that at the lower position. For the purpose of judging the results, it is assumed that the materials in the dress are same. From the experience, it can be known that the force on the shoulder is stronger than that at the bottom of the dress, because the anti-force exists on the shoulder and there is no this type of anti-force at the bottom. Thus the simulation results according to the color shown in Figure 6 can be verified.

When the displacements of all PARTs along three axes are determined, the folds of the garment can be computed along with the calculation of forces. If the difference between the resultant displacements of a PART and that of adjacent PARTs is larger, there will be more wrinkles; otherwise, there will be fewer wrinkles. With the computer simulation of the displacements within all the PARTs, the whole garment can be simulated and the details of the simulation are shown in Figure 7. The simulation procedures of different types of the garments are similar to the ones presented above.

In addition, by adjusting the lower values and upper values of distance constraints in Table 3 , the simulation results and the simulation speed can be changed. In this manner, it can be observed that the simulation time is the shortest with acceptable results, that is, proper lower values and upper values of distance constraints can bring a better performance between simulation results and simulation speed.

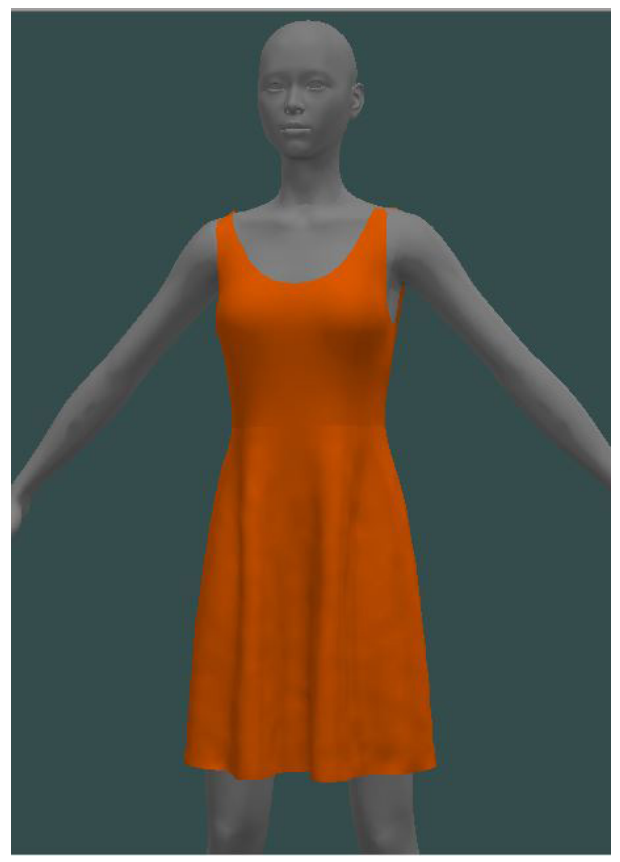

Figure 7. Dress simulation using the proposed scheme.

\section{$\underline{3.3 \text { Simulation speed comparison }}$}

With the acceptable simulation results, different methods are applied to compare the simulation speed. The methods in reference [19], [20] and [21] are chosen to be compared with the proposed scheme. In order to verify the robustness of the method, different types of the garment are used to calculate the simulation time. In the simulation, the types of garments are named as pullover 1, pullover 2, flared skirt, low-cut dress, and long-sleeved dress, whose appearances are shown in Figure 8. The computer for simulation is featured with the central processing unit (CPU) of Intel Core I5 650, 8GB memory and ATI Radeon HD5450 graphics card. The simulation times for five types of garments using different methods are listed in Table 4 and the simulation results using the proposed scheme are demonstrated in Figure 8 (a)-(e).

From Figure 8 , it is observed that the simulation results are acceptable for each type of the garment. From Table 4, it can be seen that the simulation speed of the proposed scheme is higher than that of other reference methods. For example, as for one of the different types of the garments, "Pullover 1" with 5,103 vertices, it requires about $898 \mathrm{~ms}$ when the proposed scheme is applied, while the simulation times are 1,012 , 1,133 , and $1,257 \mathrm{~ms}$, respectively, when using the methods in reference [19], [20], and [21]. Compared with the reference methods, the proposed scheme can reduce the simulation times by $11.26 \%, 20.74 \%$, and $28.56 \%$, respectively. For other types of the garments, the proposed scheme shows the smallest simulation time. Considering all the types of garments and the average simulation time of the proposed scheme, the methods in [19], [20], and [21] are, respectively, 1,105.1, $1,232.8,1,363.5$, and $1488.1 \mathrm{~ms}$. It can be concluded that the proposed scheme improves the simulation speed effectively and can help to achieve a better performance between the simulation time and the simulation accuracy.

In practice, it is natural that there are limitations in the proposed scheme. At the first stage, the distance constraints should be predefined as shown in Table 3 for the PART division. In this article, the number of levels and the lower value and the upper value of each level are given by experience initially. Then, the lower and upper values of each level are adjusted by iteration with a computer to determine the proper parameters, with which the proposed scheme requires less simulation time with acceptable simulation results. Table 3 lists one of the possible solutions suitable for the five types of garments in the simulation. The values in Table 3 may not be optimal and suitable for other types of the garments. The proposed scheme cannot provide an optimal solution for the determination of the number of the levels and range values of each level. This problem will be further studied in the future.

\section{Conclusion}

Aiming to obtain a better comprehensive performance between the simulation accuracy and the simulation time, a two-stage mechanical model for garment simulation in 3D space is proposed in this article. At the first stage, the concept PART 


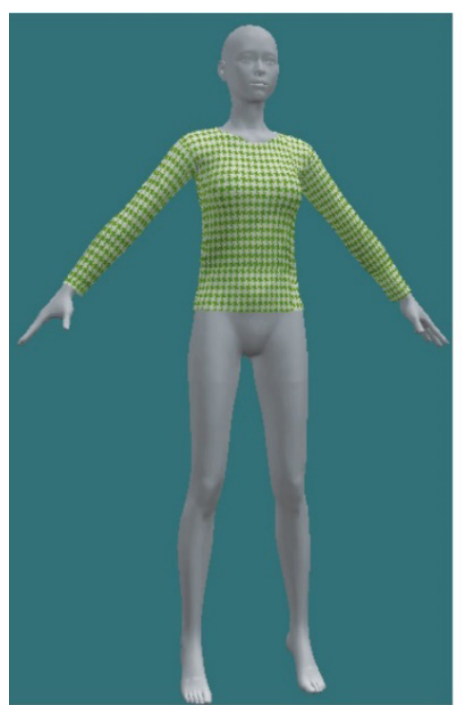

(a)

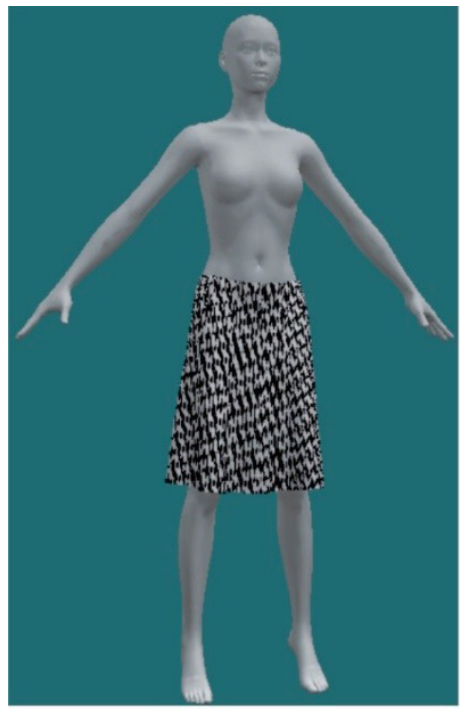

(c)

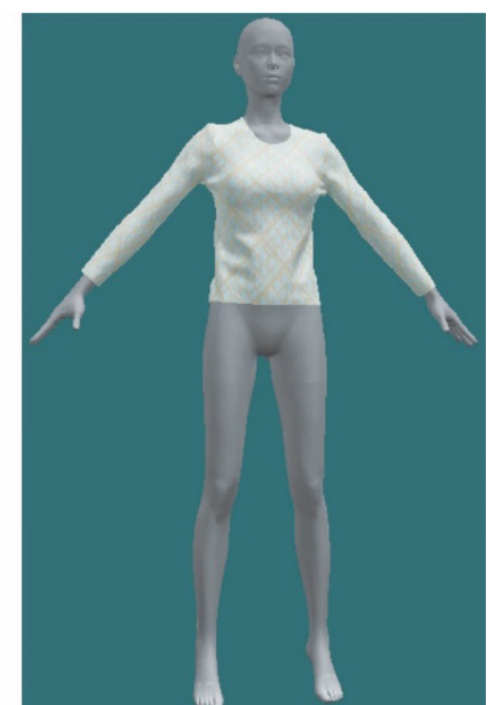

(b)

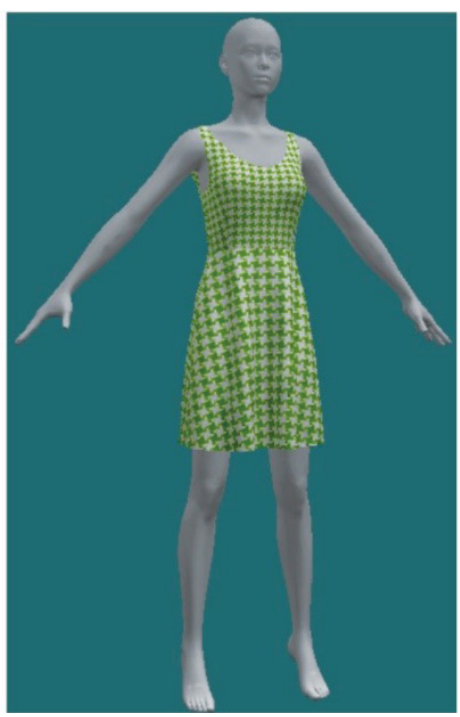

(d)

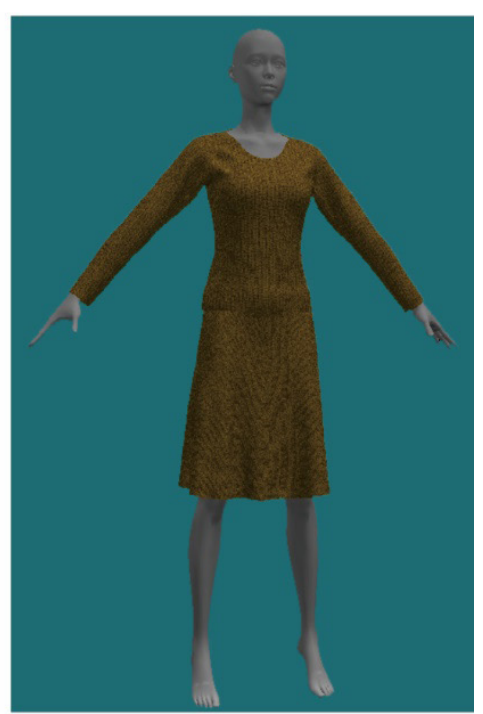

(e)

Figure 8. Different types of garment simulation with the proposed scheme.

Table 4. Simulation time comparison (in $\mathrm{ms}$ )

\begin{tabular}{|c|c|c|c|c|c|}
\hline \multirow{2}{*}{ Type } & $\begin{array}{c}\text { Number of } \\
\text { Vertices }\end{array}$ & $\begin{array}{c}\text { Method } \\
\text { in [19] }\end{array}$ & $\begin{array}{c}\text { Method } \\
\text { in [20] }\end{array}$ & $\begin{array}{c}\text { Method } \\
\text { in [21] }\end{array}$ & Proposed Method \\
\hline \multirow{2}{*}{ Pullover 1 } & 5.103 & 1.012 & 1.133 & 1.257 & 898 \\
\cline { 2 - 6 } & 4.710 & 960 & 1.084 & 1.189 & 934 \\
\hline \multirow{2}{*}{ Pullover 2 } & 4.986 & 1.002 & 1.125 & 1.198 & 912 \\
\cline { 2 - 6 } & 4.554 & 940 & 1.057 & 1.087 & 727 \\
\hline \multirow{2}{*}{ Flared skirt } & 3.920 & 859 & 965 & 998 & 1.278 \\
\hline \multirow{2}{*}{ Low-cut dress } & 3.613 & 824 & 929 & 979 & 1.196 \\
\hline \multirow{2}{*}{$\begin{array}{c}\text { Long-sleeved } \\
\text { dress }\end{array}$} & 6.463 & 1.322 & 1.454 & 1.489 & 1.823 \\
\cline { 2 - 6 } & 5.628 & 1.213 & 2.410 & 2.687 & 2.478 \\
\hline
\end{tabular}


is defined to separate the garment into several areas with the corresponding distance constraints. At the second stage, a mechanical model expressed in a general form in 3D space within a PART is derived by considering all the possible forces, including gravity and other forces. This is beneficial to the demonstration of the fold of the dress. Then, the displacements along three axes in 3D space can be computed with the proposed method and the deformation of the dress on the human body can be demonstrated. The experiment and simulation are carried out to verify the performance of the proposed scheme. The results show that the proposed scheme can improve the simulation speed effectively with acceptable results. In practice, the proposed scheme has limitations in the determination of the distance constraints, including the number of levels and the range values at the first stage. In this article, the distance constraints are obtained by iteration with the computer starting from the initial distance constraints provided by experience. The distance constraints may not be optimal. The problem of how to design the optimal distance constraints will be studied in the future.

\section{Acknowledgments}

The authors acknowledge the financial support from the Standard and mode of intelligent manufacturing, Ministry of Industry and Information Technology of the People's Republic of China ([2016]213); National Science Foundation of China (61772238); and a project funded by the Priority Academic Program Development of Jiangsu Higher Education Institutions (PAPD, No. [2014]37).

\section{References}

[1] P., Volino, N. Magnenat-Thalmann. Virtual garment, theory and practice. Springer 2000.

[2] De Aguiar E, et al. Stable spaces for real-time garment [J]. ACM Transactions on Graphics, 2010, 29 (4):106.

[3] Wang $H$, Hecht $F$ et al. Example-based wrinkle synthesis for garment animation [J]. ACM Transactions on Graphics, 2010, 29(4):101-107.

[4] Choi, K., And Lo, T. An energy model of plain knitted fabric. [J]. Textile Research Jour. 2003, 73:739-748.

[5] Gabriel Cirio, Jorge Lopez-Moreno Efficient Simulation of knitted garment using persistent contacts[C]. SCA'S15 Proceedings of the 14th ACM SIGGRAPH 201555-61.
[6] Kaldor J.M, James D.L., And Marschner S .Efficient yarnbased garment with adaptive contact linearization. [C] ACM T. Graph.(SIGGRAPH 10)29,4,105

[7] $X$. Provot. Deformation constraints in a mass-spring mode to describe rigid garment behavior [C]. Proc. of Graphics Interface, Quebec, 1995, 147-154.

[8] Terzopoulos D, Platt J, Barr A, Fleischer K. Elastically deformable models [C].Computer Graphics (SIGGRAPH'87), 1987, 21(4):205-214

[9] M. Carignan, Y. Yang, N.M. Thalmann, and D. Thalmann. Dress Animated synthetic actors with complex deformable garments. [J]. Computer Graphics, 1992,26(2): 99-104

[10] Volino P, Magnenat-Thalmann N. Virtual garment-theory and practice.[M].2000.

[11] Baraff D, Witkin A. Large steps in garment simulation [C]. Proceedings of the 25th Annual conference on Computer Graphics and Interactive Techniques. New York; ACM Press. 1988:43-54.

[12] Hadap S, Bangerter E, Volino P, Magnenat-Thalmann N. Animating wrinkles on clothes. In Proc. of the Conf. on Visualization'99:Celebrating Ten Years. IEEE Computer Society Press, 1999.175-182.

[13] K.J. Choi, H.S. Ko. Stable but responsive garment. [J]. ACM Transactions on Graphics.2002, 21(3): 604-611.

[14] Y.M. Kang, H.G. Cho. Bilayered approximate integration for rapid and plausible animation of virtual garment with realistic wrinkles. [J]. Proceedings of Computer.2001, 17(3):147-157.

[15] F. Cordier, N. Magnenat-Thalmann. Real-time animation of dressed virtual humans. [J].Computer Graphics Forum.2002,21(3): 327-336.

[16] Liu Jiongzhou, Li Jituo, Lu Guodong. Physicalgeometric mixed model for $3 D$ clothing simulation [J]. Journal of Computer-Aided Design \& Computer Graphics.2014,26(12): 2245-2250.

[17] Yang Jian dong, Shang Shuyuan. Garment modeling simulation based on mass spring model [J]. Applied Mechanics, 2013, 310:676-683

[18] Muller M, Chentanez N. Wrinkle meshes [C].Proceedings of the 2010 ACM

[19] Dongyong Zhu, Zhong Li. Dynamic Garment simulation based on hybrid bounding volume hierarchy [J]. AUTEX Research Journal, Vol. 19, No 1, March 2019, 2016, 16(4):241-249.

[20] Clemmens Groß Automatic Pre-positioning of virtual clothing[J].Spring Conference on computer graphics ,2003,99-108

[21] A Consistent bending model for cloth simulation with corotational subdivision finite elements[J].SCA'06 Proceedings of the 2006 ACM SIGGRAPH/Eurographics symposium on Computer animation, 2006,107-116 